Rev. Int. Contam. Ambie. 36 (1) 45-53, 2020

DOI: $10.20937 /$ RICA.2020.36.53391

\title{
VALIDACIÓN DE UNA METODOLOGÍA PARA LA DETERMINACIÓN DE As(III) Y As(V) EN AGUAS DE CONSUMO HUMANO POR CROMATOGRAFÍA DE INTERCAMBIO ANIÓNICO Y ESPECTROMETRÍA DE MASAS CON PLASMA DE ACOPLAMIENTO INDUCTIVO
}

\author{
Validation of a methodology to determine $\mathrm{As}(\mathrm{III})$ and $\mathrm{As}(\mathrm{V})$ in drinking water by \\ anion exchange chromatography and inductively coupled plasma mass spectrometry
}

Betzabel ARIAS-BARRANTES ${ }^{1 *}$, Azucena URBINA-CAMPOS ${ }^{1}$ y Ana Lorena ALVARADO-GÁMEZ ${ }^{2}$

${ }^{1}$ Laboratorio Nacional de Aguas, Instituto Costarricense de Acueductos y Alcantarillados, A.P. 1097-1200, San José, Costa Rica

${ }^{2}$ Centro de Investigación en Contaminación Ambiental, docente en la Escuela de Química de la Universidad de Costa Rica, A.P. 11501-2060, San José, Costa Rica

*Autora para correspondencia: bearias@aya.go.cr

(Recibido: agosto 2018; aceptado: marzo 2019)

Palabras clave: arsénico, arsenito, arseniato, arsénico soluble, arsénico particulado, ICP-MS, estados de oxidación

\section{RESUMEN}

Se optimizó y validó una metodología para la determinación de arsénico soluble y particulado (As[III] y As[V]) en aguas de consumo humano (ACH) empleando cromatografía de intercambio aniónico (CIA) y espectrometría de masas con plasma de acoplamiento inductivo (ICP-MS). La optimización de CIA consistió en una simulación en el laboratorio del procedimiento a seguir en el campo, donde se evaluó la recuperación de 10 mezclas de disoluciones de As(III)/As(V) en agua sintética. Se obtuvieron las siguientes figuras de mérito en el análisis de arsénico total: límite de detección (LD) de $0.1 \mu \mathrm{g} / \mathrm{L}$, límite de cuantificación (LC) de $0.2 \mu \mathrm{g} / \mathrm{L}$, ámbito lineal de $0.1 \mu \mathrm{g} / \mathrm{L}$ a $300 \mu \mathrm{g} / \mathrm{L}$ y coeficientes de determinación $\leq 0.9996$. La repetibilidad y repetibilidad intermedia fueron evaluadas mediante la lectura de un patrón de $12.5 \mu \mathrm{g} / \mathrm{L}$ de arsénico total: el promedio de repetibilidad fue de $12.6 \mu \mathrm{g} / \mathrm{L}$ con una desviación estándar $\left(\mathrm{S}_{0}\right)$ de $0.2 \mu \mathrm{g} / \mathrm{L}$ y un coeficiente de variación $(\mathrm{CV})$ de $0.2 \%$ para $\mathrm{n}=7$, mientras que la repetibilidad intermedia tuvo un promedio de $12.6 \mu \mathrm{g} / \mathrm{L}$ con $\mathrm{S}_{0}=0.4 \mu \mathrm{g} / \mathrm{L}$ y un CV de $0.3 \%$ para $\mathrm{n}=7$. Las recuperaciones promedio fueron: arsénico total $\leq 99 \%$, arsénico soluble $\leq 99 \%$, y arsenito y arseniato $\leq 97 \%$.

Key words: arsenic, arsenite, arsenate, soluble arsenic, particulate arsenic, ICP-MS, oxidation states

\begin{abstract}
A methodology was optimized and validated for the determination of soluble and particulate arsenic (As[III] and As[V]) in drinking water using anion exchange chromatography (AEC) and inductively coupled plasma mass spectrometry (ICP-MS). The AEC optimization consisted in a laboratory simulation of the procedure to be followed in the field to evaluate the recovery of 10 mixtures of $\mathrm{As}(\mathrm{III})$ and $\mathrm{As}(\mathrm{V})$ in synthetic water. The following figures of merit were obtained in the total arsenic analysis: detection
\end{abstract}


limit (LOD) of $0.1 \mu \mathrm{g} / \mathrm{L}$, quantification limit (LOQ) of $0.2 \mu \mathrm{g} / \mathrm{L}$, linear range between $0.1 \mu \mathrm{g} / \mathrm{L}$ and $300 \mu \mathrm{g} / \mathrm{L}$, and coefficients of determination $\leq 0.9996$. Repeatability and intermediate repeatability were evaluated with a total arsenic standard solution of $12.5 \mu \mathrm{g} / \mathrm{L}$, obtaining an average repeatability of $12.6 \mu \mathrm{g} / \mathrm{L}$ with a standard deviation of $0.2 \%$ and a coefficient variation of $0.2 \%$ for $n=7$, while the intermediate precision had an average of $12.6 \mu \mathrm{g} / \mathrm{L}$ with a standard deviation of $0.4 \%$ and a coefficient variation of $0.3 \%$ for $n=7$. The average recoveries in the mixtures were: total arsenic $\leq 99 \%$, soluble arsenic $\leq 99 \%$ and for arsenite and arsenate $\leq 97 \%$.

\section{INTRODUCCIÓN}

Los dos estados de oxidación del arsénico mayoritarios en la naturaleza son el arsenito o As(III) y el arseniato o $\mathrm{As}(\mathrm{V})$. Ambos se encuentran en la fracción soluble del arsénico en el agua (Akter et al. 2005, Ahmed et al. 2010). Conocer las concentraciones de arsenito y arseniato es fundamental para la interpretación de la toxicidad, movilidad y transformación geoquímica del arsénico en el ambiente pues la toxicidad del As(III) es mayor que la del $\mathrm{As}(\mathrm{V})$, debido a que se bioacumula de manera distinta (Wang y Wai 2004, Haxton 2011). Desde el punto de vista de la remediación, se aporta valiosa información sobre la tecnología de remoción más apropiada, pues es más difícil la remoción de As(III) que de As(V) (Sharma y Sohn 2009, Moreno-Jiménez 2010).

La determinación analítica de As(III) y As(V) es una valiosa herramienta de investigación, con la cual se pueden separar y cuantificar estas especies arsenicales presentes en aguas a nivel de trazas y ultratrazas (Aposhian y Aposhian 2006). Debe considerarse que uno de los métodos debe facilitar el procedimiento de separación, el cual debe ser eficaz y fiable, mientras que la otra técnica debe proporcionar la detección adecuada así como la cuantificación de las especies de interés (Smedley y Kinniburgh 2002, Bednar et al. 2004, Voice et al. 2011).

Por lo general, en la determinación de especies arsenicales o estados de oxidación se emplea una técnica cromatográfica acoplada con espectrometría atómica o ICP-MS, o bien, técnicas electroquímicas que resultan atractivas desde el punto de vista económico y analítico (Akter et al. 2005, Hagiwara et al. 2015).

Al realizar la separación y cuantificación de los estados de oxidación del arsénico, se debe considerar la posible modificación de las especies químicas en el proceso de almacenamiento y transporte de la muestra hasta el laboratorio para su posterior análisis, ya que tanto el As(III) como el As(V) tienden a ser inestables después de que se separan de su entorno químico y biológico (McCleskey et al. 2004, Voice et al. 2011).

Se puede optar por un pre-tratamiento de las muestras y asegurarse un mayor tiempo de estabilidad de las especies, sin embargo, existen discrepancias en la literatura publicada sobre cuál es el mejor método para preservar el arsenito y el arseniato (Bednar et al. 2002, McCleskey et al. 2004, Kumar y Riyazuddin 2010).

Debido a la nula existencia de un método universal que asegure la correcta preservación, es preferible efectuar la separación con cromatografía de intercambio aniónico (CIA) en el momento de recolección de la muestra, con el fin de asegurar que las especies que se encuentran en el ambiente acuático natural no sufran transformación alguna, lo cual hace que la cuantificación del arsenito y arseniato sea mucho más exacta (Voice et al. 2011, Sorg et al. 2014).

Para preservar las muestras se emplea ácido nítrico grado ultrapuro, ácido clorhídrico o sulfúrico de la misma calidad, o bien ácido etilendiaminotetraacético (EDTA). La escogencia del preservante depende del tipo de muestra, análisis, o especies químicas a cuantificar (Akter et al. 2005, Voice et al. 2011). Después de la acidificación, es recomendable el transporte de las mismas a $4{ }^{\circ} \mathrm{C}$ para evitar la alteración del volumen recolectado por evaporación (Bednar et al. 2004). Además, se aconseja el empleo de botellas de polipropileno opacas para disminuir reacciones químicas indeseables y actividad microbiana por la luz del sol (Litter et al. 2009).

El objetivo de esta investigación es optimizar y validar una metodología analítica a partir de cromatografía de intercambio aniónico y espectrometría de masas con plasma de acoplamiento inductivo para cuantificar arsénico total, arsénico soluble y arsénico particulado (As[III] y As[V]) en aguas sin cloración. 


\section{MATERIALES Y MÉTODOS}

\section{Reactivos}

Reactivos estándar de 22 elementos de calibración para espectrometría de masas con plasma de acoplamiento inductivo (ICP-MS, por sus siglas en inglés) de $10 \mu \mathrm{g} / \mathrm{mL}$ (método 6020A de Accustandard) y que contiene arsénico cuya concentración es comparada contra un material de referencia del National Institute of Standards and Technology (NIST; EUA), sal de arsenito de sodio $\left(\mathrm{AsNaO}_{2}\right)$, pureza $\geq 90 \%$, sal de arseniato de sodio dibásico heptahidratado $\left(\mathrm{Na}_{2} \mathrm{HAsO}_{4}\right)$, pureza $\geq 99.995 \%$, estándar de As(III) de $1000 \mathrm{mg} / \mathrm{L}$ para ICP-MS de Sigma-Aldrich, estándar de As(V) de $1000 \mathrm{mg} / \mathrm{L}$ para ICP de Sigma-Aldrich, resina Dowex 1-X8de 50a 100mallas, $\mathrm{NaOHde} \mathrm{1.0} \mathrm{mol/L,ácidoacético}$ $1.0 \mathrm{~mol} / \mathrm{L}$, ácido nítrico al $70 \%$ ultrapuro, ácido sulfúrico al $5 \%$, ultrapuro, agua desionizada, disoluciones de trabajo para ICP-MS: disolución de sintonización de tecnologías Agilent para ICP-MS de $1 \mu \mathrm{g} / \mathrm{L}$ de concentración final en los siguientes elementos: $\mathrm{Li}, \mathrm{Tl}$, Y, Ce y Co, disolución estándar interno para ICP-MS de Agilent Technologies de $400 \mu \mathrm{g} / \mathrm{L}$ de concentración final en los siguientes elementos: $\mathrm{Bi}, \mathrm{Ge}, \mathrm{In}, \mathrm{Li}, \mathrm{Lu}$, $\mathrm{Rh}$, Sc y Tb, disoluciones de sintonización 1 y 2 de Agilent Technologies para verificación de factor P/A (factor pulso - analógico) con más de 33 elementos en concentraciones desde $5 \mu \mathrm{g} / \mathrm{L}$ hasta $40 \mu \mathrm{g} / \mathrm{L}$.

\section{Materiales}

Tres botellas de polietileno de alta densidad certificadas para metales de $250 \mathrm{~mL}$ de capacidad rotuladas como A, B y C, columnas cromatográficas de polipropileno de $20 \mathrm{~mL}$, jeringas plásticas de $50 \mathrm{~mL}$, filtros de disco de $0.45 \mu \mathrm{m}$ de nylon, probeta de $50 \mathrm{~mL}$ preferiblemente plástica, vaso de plástico de dos litros, tubos de ensayo de ensayo de polipropileno.

\section{Optimización del equipo ICP-MS}

Se empleó el ICP-MS marca Agilent Technologies, serie 7700. Este equipo posee un sistema de introducción de muestra que consiste en una cámara de espray de doble paso hecha de cuarzo y un nebulizador tipo Micromist, lentes iónicos tipo " $\mathrm{x}$ ", un sistema de reacción octapolar entre las lentes y cuadrupolo para eliminar interferencias poliatómicas. . El cuadrupolo de este equipo tiene un prefiltro y luego un filtro principal, mientras que el detector es un multiplicador de electrones que opera en modo pulso y analógico. La optimización del método se efectuó de acuerdo a las especificaciones del manual del fabricante (Agilent Technologies 2011) y consistió en dos pasos: primero la verificación de parámetros comunes del sistema como comprobación general (cuadro I) empleando la disolución de sintonización de Agilent Technologies de $1 \mu \mathrm{g} / \mathrm{L}$ de concentración así como las disoluciones de sintonización de factor P/A del mismo fabricante. El segundo paso es la calibración de masas con la disolución sintonizadora y la disolución de patrón interno de $400 \mu \mathrm{g} / \mathrm{L}$. En la calibración de masas se monitorean las cuentas para ${ }^{59} \mathrm{Co}$ (superiores a $1900 \mathrm{cps}$ ), ${ }^{159} \mathrm{~Tb}$ (cuentas superiores a $178000 \mathrm{cps}$ ) ${ }^{205} \mathrm{Tl}$ (cuentas superiores

CUADRO I. PARÁMETROS DE COMPROBACIÓN INICIAL DIARIOS VERIFICADOS EN EL INICIO

\begin{tabular}{lll}
\hline Parámetro & Masas monitoreadas & Objetivo \\
\hline Eje de la antorcha & ${ }^{7} \mathrm{Li},{ }^{59} \mathrm{Co},{ }^{205} \mathrm{Tl}$ & $\begin{array}{l}\text { Posición óptima de la antorcha alineada con la interfase: distancia inyector/ } \\
\text { antorcha/conos }\end{array}$ \\
\hline Espectrómetro de masas & $\begin{array}{l}\text { Corrección de las posibles diferencias que pueden existir entre la masa leída } \\
\text { por el equipo y la masa exactamente conocida para cada isótopo }\end{array}$ \\
\hline Corrección del plasma & $\begin{array}{l}\text { Corregir posibles fluctuaciones que se presentan en el plasma y que puedan } \\
\text { afectar la precisión de la medida }\end{array}$ \\
\hline $\begin{array}{l}\text { Sintonización estándar de } \\
\text { las lentes }\end{array}$ & ${ }^{7} \mathrm{Li},{ }^{59} \mathrm{Co},{ }^{205} \mathrm{Tl}$ & $\begin{array}{l}\text { Las lentes de extracción y lente iónica deben estar en posición fuera de eje, } \\
\text { asegurando una alta transmisión iónica en todo el rango de masas }\end{array}$ \\
\hline $\begin{array}{l}\text { Resolución } \\
\text { del eje }\end{array}$ & ${ }^{7} \mathrm{Li},{ }^{59} \mathrm{Co},{ }^{205} \mathrm{Tl}$ & Mejor balance entre la separación de los picos y la relación señal/ruido \\
\hline $\begin{array}{l}\text { Reporte } \\
\text { de ejecución }\end{array}$ & $\begin{array}{l}\text { Óxidos: } 140 \\
\mathrm{Cargas} \text { dobles: } 140\end{array}$ & $\begin{array}{l}\text { La formación de óxidos y cargas dobles debe ser menor al 2 \%. Los óxidos } \\
\text { se monitorean en el par CeO/Ce }\end{array}$ \\
\hline $\begin{array}{l}\text { Factor P/A } \\
\text { (modo pulso/analógico) }\end{array}$ & $\begin{array}{l}7,23,27,88,89,95,101, \\
105,111,115,118 \text { y 205 }\end{array}$ & $\begin{array}{l}\text { Efectúa la calibración en modo pulso y analógico del detector de manera si- } \\
\text { multánea. Este factor es empleado para convertir un valor del modo analógico } \\
\text { en uno para el modo de pulso }\end{array}$ \\
\hline
\end{tabular}


a $3000 \mathrm{cps}$ ). Ambos procedimientos se efectuaron diariamente, antes de iniciar la medición de los patrones de la curva de calibración de arsénico, los controles de calidad y las muestras.

Se trabajó con el isótopo ${ }^{75} \mathrm{As}$, mientras que la interferencia espectral ${ }^{40} \mathrm{Ar}{ }^{35} \mathrm{Cl}$ se evitó por medio el sistema de colisión octapolar con gas helio que suprime esta interferencia mediante discriminación de energía cinética (modo KED, por sus siglas en inglés).

Se empleó germanio como estándar interno y se obtuvo una recuperación de $70 \%$ a $125 \%$. Por otra parte, se empleó el estándar de control de calidad 2 para ICP-MS de Accustandard, diluido a $50 \mu \mathrm{g} / \mathrm{L}$ como concentración final y debió recuperarse entre $90 \%$ y $110 \%$, según lo indicado en los métodos estándar para la determinación de agua y agua residual de la American Public Health Association (APHA 2012).

\section{Optimización de la cromatografía de intercambio aniónico \\ Preparación de resina de intercambio aniónico en forma de acetato}

Se siguió el procedimiento descrito por la Agencia de Protección del Medio Ambiente de EUA (USEPA, por sus siglas en inglés; 2000). Se pesaron $200 \mathrm{~g}$ de resina Dowex 1-X8 de 50 a 100 mallas en un vaso de precipitados plástico y se adicionaron 200 $\mathrm{mL}$ de $\mathrm{NaOH} 1.0 \mathrm{~mol} / \mathrm{L}$. Se colocó en una plantilla de agitación constante en capilla de extracción por una hora, se dejó reposar 5 min y luego se decantó el $\mathrm{NaOH}$. Este lavado se repitió dos veces más. La resina se lavó dos veces con $200 \mathrm{~mL}$ de agua desionizada. Posteriormente, se lavó tres veces con ácido acético $1.0 \mathrm{~mol} / \mathrm{L}$ empleando agitación constante por $5 \mathrm{~min}$. Finalmente, se efectuaron tres lavados de la resina con agua desionizada antes de ser empacada.

La columna cromatográfica de polipropileno se enjuagó previamente con agua desionizada y se dejó secar a temperatura ambiente. Se empacaron $20 \mathrm{~g}$ de resina (peso escurrido) en cada columna, dejando aproximadamente $1 \mathrm{~cm}$ de altura de agua desionizada para mantener la resina húmeda.

\section{Preparación de disoluciones de As(III) y As(V) en agua sintética}

Para la optimización de las columnas de intercambio aniónico, se realizó una simulación en el laboratorio del procedimiento a efectuar en el campo descrito por la USEPA (2000), empleando muestras de agua sintética (conductividad inferior a $1 \mu \mathrm{S} / \mathrm{cm}$ ) enriquecidas con concentraciones conocidas de $\mathrm{As}(\mathrm{III})$ y As(V), para verificar que la resina retuviese efectivamente el $\mathrm{As}(\mathrm{V})$ presente como oxianión y así evaluar la recuperación de arsenito y arseniato después del tratamiento en la columna.

Las disoluciones de $\mathrm{As}$ (III) se prepararon a partir de $\mathrm{AsNaO}_{2}$ y las de $\mathrm{As}(\mathrm{V})$ a partir de $\mathrm{Na}_{2} \mathrm{HAsO}_{4}$. Las disoluciones intermedias y las de trabajo (concentración final de $500 \mu \mathrm{g} / \mathrm{L}$ ) se guardaron en botellas de polietileno de alta densidad color ámbar y en refrigeración.

Se prepararon 10 mezclas de As(III) / As(V), cuyau composición se detalla en el cuadro II. Se trabajó en dos ámbitos de concentración: ámbito alto en concentraciones de $50.0 \mu \mathrm{g} / \mathrm{L}$ y $25.0 \mu \mathrm{g} / \mathrm{L}$, y ámbito bajo a $5.0 \mu \mathrm{g} / \mathrm{L}$ de As(III) y As(V) y en ausencia de As(III) o As(V). Se seleccionaron estos ámbitos con el fin de no saturar la resina, ya que una vez validado el método, éste se emplearía para especiar agua con concentraciones de arsénico inferiores a $10.0 \mu \mathrm{g} / \mathrm{L}$ y hasta los $50.0 \mu \mathrm{g} / \mathrm{L}$ como máximo.

CUADRO II. DIEZ MEZCLAS DE As(V) Y As(III) PARA EVALUAR LA RECUPERACIÓN DEL MÉTODO

\begin{tabular}{ccc}
\hline Mezcla & $\begin{array}{c}\text { Concentración en } \\
\text { As }(\mathrm{V})(\mu \mathrm{g} / \mathrm{L})\end{array}$ & $\begin{array}{c}\text { Concentración en } \\
\text { As }(\mathrm{III})(\mu \mathrm{g} / \mathrm{L})\end{array}$ \\
\hline 1 & 25.0 & 5.0 \\
2 & 0 & 5.0 \\
3 & 5.0 & 0 \\
4 & 50.0 & 5.0 \\
5 & 25.0 & 25.0 \\
6 & 50.0 & 0 \\
7 & 0 & 50.0 \\
8 & 25.0 & 0 \\
9 & 5.0 & 50.0 \\
10 & 5.0 & 25.0 \\
\hline
\end{tabular}

Para cada mezcla se llevaron a cabo siete repeticiones. Una vez preparada la mezcla, se realizó el procedimiento de filtración y el tratamiento en la columna cromatográfica de forma inmediata para evitar la posible oxidación del arsenito.

\section{Procedimiento de filtración y separación}

A la botella A se le adicionaron $0.5 \mathrm{~mL}$ de $\mathrm{HNO}_{3}$ concentrado grado ultrapuro al $70 \%$ y se llenó con $250 \mathrm{~mL}$ de la mezcla preparada. Se cerró la botella y se colocó papel parafilm alrededor de la tapa para evitar contaminación hasta su análisis. 
La jeringa de $50 \mathrm{~mL}$ se lavó tres veces con el agua restante de la mezcla preparada, procurando no agregar aire a la hora del lavado y toma de muestra. Después se llenó y se colocó en ella el filtro de 0.45 $\mu \mathrm{m}$. Se descartaron las primeras diez gotas que pasaron por el filtro.

Después de lavar dicho filtro, se tomó la botella $\mathrm{B}$, la cual contenía $1.25 \mathrm{~mL}$ de $\mathrm{H}_{2} \mathrm{SO}_{4}$ ultra puro al $5 \%$ y se llenó con la muestra de la mezcla ya filtrada (arsénico soluble). Se repitió el procedimiento hasta recolectar $125 \mathrm{~mL}$. Se cerró la botella y se agitó vigorosamente por $15 \mathrm{~s}$.

Se tomó una de las columnas y antes de pasar la muestra filtrada (botella B), se descartó el agua desionizada que mantenía la resina húmeda.

La muestra filtrada se hizo pasar por la columna. Los primeros $40 \mathrm{~mL}$ que se eluyeron de la columna se midieron con una probeta y se descartaron con el fin de lavar la columna con la muestra varias veces hasta recolectar la fracción final para análisis. Finalmente, se colocó la columna en la botella $\mathrm{C}$ la cual contenía una gota de $\mathrm{HNO}_{3}$ concentrado ultrapuro y se recolectaron aproximadamente $20 \mathrm{~mL}$ del agua tratada por la resina (la fracción recolectada corresponde al As[III] mientras que lo que quedó retenida en la columna corresponde al As[V]). Las botellas B y C se taparon y se sellaron las tapas con papel parafilm.

\section{Cuantificación en el laboratorio mediante ICP-MS}

Cabe destacar que una vez separadas y preservadas las distintas formas de arsénico en campo, las muestras pueden almacenarse hasta por seis meses empleando los recipientes adecuados y la cantidad de ácido ultrapuro apropiada (Bednar et al. 2002, Kumar y Riyazuddin 2010).

Antes de que la muestra se tomara definitivamente para su lectura, se enjuagó con ésta tres veces un tubo de ensayo de polipropileno, el cual después se colocó en el automuestreador del ICP-MS. A partir de las muestras $\mathrm{A}, \mathrm{B}$ y $\mathrm{C}$ se obtuvieron los resultados de arsénico total, arsénico soluble, arsénico particulado, As(III) y As(V). En el cuadro III se resume el contenido analizado de cada una de las tres botellas.

\section{Validación de la metodología CIA/ICP-MS}

Para el tratamiento y evaluación de los datos destinados a la validación de la metodología CIA/ ICP-MS se siguió lo establecido por la Guía Eurachem 2012 (Ellison y Williams 2012). Las figuras de mérito determinadas fueron el límite de detección y el límite de cuantificación instrumentales, calculados de acuerdo con lo establecido por Currie (1995) y Miller y Miller (2002). Se determinó la desviación estádar $\left(\mathrm{S}_{\mathrm{B} 0}\right)$ de siete repeticiones de la intensidad del blanco y se consideró el límite de detección (LD) como $3 \times \mathrm{S}_{\mathrm{B} 0} / \mathrm{m}$, siendo $\mathrm{m}$ la pendiente de la curva de calibración; asimismo, se consideró el límite de cuantificación (LC) como $10 \times \mathrm{S}_{\mathrm{B} 0} / \mathrm{m}$. Por otra parte, los límites de detección y cuantificación del método se calcularon determinando la desviación estándar de la medición de 10 patrones de arsénico de $1.0 \mu \mathrm{g} / \mathrm{L} \mathrm{a}$ partir del material de referencia; se consideró el LD como $3 \times \mathrm{S}_{0}$ y el LC como $10 \times \mathrm{S}_{0}$.

La repetibilidad se calculó midiendo siete veces un patrón de arsénico de $12.5 \mu \mathrm{g} / \mathrm{L}$. A partir de este mismo patrón y con la misma cantidad de lecturas pero en distintas condiciones ambientales e instrumentales (diferentes días), se estimó la precisión intermedia.

La veracidad se determinó con las muestras de agua sintética enriquecidas con $\mathrm{As}(\mathrm{III})$ y $\mathrm{As}(\mathrm{V})$ después de la separación con CIA y posteriormente se analizaron las muestras por ICP-MS para determinar los porcentajes de recuperación (Cuadro IV).

La linealidad se evaluó a partir del coeficiente de correlación de las distintas curvas de calibración

CUADRO III. DESCRIPCIÓN DEL CONTENIDO DE LAS BOTELLAS DE RECOLECCIÓN DE MUESTRAS DE AGUAS PARA LA SEPARACIÓN Y CUANTIFICACIÓN DE ARSÉNICO SOLUBLE, ARSÉNICO PARTICULADO, As(III) Y As(V)

\begin{tabular}{lllll}
\hline Etiqueta & $\begin{array}{l}\text { Cantidad de } \\
\text { muestra }(\mathrm{mL})\end{array}$ & Preservación & $\begin{array}{l}\text { Muestra } \\
\text { contenida }\end{array}$ & $\begin{array}{l}\text { Tipo de arsénico a } \\
\text { cuantificar }\end{array}$ \\
\hline A & 250 & $\begin{array}{l}\mathrm{HNO}_{3} \text { concentrado } \\
\text { ultra puro } \mathrm{pH}<2\end{array}$ & $\begin{array}{l}\text { Agua de la fuente } \\
\text { de captación }\end{array}$ & $\begin{array}{l}\text { Arsénico } \\
\text { total }\end{array}$ \\
\hline B & 125 & $\begin{array}{l}\mathrm{H}_{2} \mathrm{SO}_{4} 5 \% \\
\text { ultra puro } \mathrm{pH} \approx 2\end{array}$ & $\begin{array}{l}\text { Agua filtrada } \\
\text { y acidificada }\end{array}$ & $\begin{array}{l}\text { Arsénico soluble y } \\
\text { particulado (por diferencia) }\end{array}$ \\
\hline C & 20 & $\begin{array}{l}\mathrm{HNO}_{3} \text { concentrado } \\
\text { ultra puro } \mathrm{pH}<2\end{array}$ & $\begin{array}{l}\text { Agua filtrada } \\
\text { y tratada por la resina }\end{array}$ & $\begin{array}{l}\text { As(III) y As(V) } \\
\text { (por diferencia) }\end{array}$ \\
\hline
\end{tabular}


CUADRO IV. RESUMEN DE LAS RECUPERACIONES PROMEDIO DE LAS MEZCLAS DE As(III) Y As(V) PARA LA OPTIMIZACIÓN DE CROMATOGRAFÍA DE INTERCAMBIO ANIÓNICO

\begin{tabular}{|c|c|c|c|c|c|c|c|c|c|c|c|c|}
\hline \multirow{2}{*}{$\begin{array}{c}\text { Mezcla } \\
\begin{array}{c}\mathrm{As}(\mathrm{V}) / \mathrm{As}(\mathrm{III}) \\
(\mu \mathrm{g} / \mathrm{L})\end{array}\end{array}$} & \multicolumn{3}{|c|}{ Recuperación de As total } & \multicolumn{3}{|c|}{ Recuperación de As soluble } & \multicolumn{3}{|c|}{ Recuperación de As(V) } & \multicolumn{3}{|c|}{ Recuperación de As(III) } \\
\hline & $\bar{X}$ & $\mathrm{~S}_{0}$ & $\mathrm{CV}(\%)$ & $\overline{\mathrm{X}}$ & $\mathrm{S}_{0}$ & $\mathrm{CV}(\%)$ & $\overline{\mathrm{X}}$ & $\mathrm{S}_{0}$ & $\mathrm{CV}(\%)$ & $\overline{\mathrm{X}}$ & & $\mathrm{CV}(\%)$ \\
\hline 25.0 y 5.0 & 101 & 2.6 & 2.6 & 101 & 2.3 & 2.3 & 100 & 2.5 & 2.5 & 103 & 9.6 & 9.3 \\
\hline 0 y 5.0 & 104 & 7.3 & 7.0 & 101 & 7.9 & 7.8 & NA & NA & NA & 97 & 10.5 & 10.8 \\
\hline 5.0 у 0 & 99 & 5.3 & 5.4 & 100 & 5.3 & 5.3 & 97 & 5.7 & 5.9 & NA & NA & NA \\
\hline 50.0 y 5.0 & 101 & 1.2 & 1.2 & 101 & 0.9 & 0.9 & 100 & 1.1 & 1.1 & 105 & 8.8 & 8.4 \\
\hline 25.0 y 25.0 & 99 & 1.0 & 1.0 & 99 & 0.6 & 0.6 & 99 & 3.0 & 3.0 & 99 & 2.9 & 2.9 \\
\hline 50.0 у 0 & 100 & 1.1 & 1.1 & 100 & 1.2 & 1.2 & 100 & 1.1 & 1.1 & NA & NA & NA \\
\hline 0 у 50.0 & 99 & 1.9 & 1.9 & 99 & 1.6 & 1.6 & NA & NA & NA & 99 & 1.7 & 1.7 \\
\hline 25.0 y 0 & 99 & 2.5 & 2.5 & 100 & 1.5 & 1.5 & 99 & 1.5 & 1.5 & NA & NA & NA \\
\hline 5.0 y 50.0 & 100 & 1.9 & 1.9 & 100 & 1.5 & 1.5 & 103 & 13.2 & 12.8 & 100 & 1.9 & 1.9 \\
\hline 5.0 y 25.0 & 104 & 7.6 & 7.3 & 99 & 2.9 & 2.9 & 97 & 5.1 & 5.3 & 102 & 2.6 & 2.5 \\
\hline
\end{tabular}

Incertidumbre de la concentración As: $\pm 0.1 \mu \mathrm{g} / \mathrm{L}$

NA: no aplica

obtenidas en cada lectura. Las concentraciones de los siete patrones de arsénico fueron: 0, 0.5, 5.0, 12.5, $25.0,50.0,100.0$ y $300.0 \mu \mathrm{g} / \mathrm{L}$.

\section{RESULTADOS Y DISCUSIÓN}

En el cuadro $\mathbf{V}$ se resumen las condiciones de lectura promedio que presentó el ICP-MS en la medición de las muestras. El patrón interno recuperado fue de 99 a $102 \%$. Los porcentajes de desviación estándar relativa fueron inferiores al $5 \%$. Las curvas de calibración con las que se midieron las muestras tuvieron coeficientes de correlación iguales o superiores a 0.9996 (Fig. 1).

La repetibilidad y la repetibilidad intermedia para siete repeticiones tuvieron un coeficiente de variación (CV) de $0.2 \%$ y de $0.3 \%$, respectivamente. Los límites de detección y cuantificación instrumentales fueron 0.02 y $0.03 \mu \mathrm{g} / \mathrm{L}$, respectivamente, mientras que los límites de detección y cuantificación del método fueron 0.1 y $0.2 \mu \mathrm{g} / \mathrm{L}$, respectivamente, expresados como arsénico total.

Los resultados de las recuperaciones promedio del arsénico total, soluble y particulado, así como de $\mathrm{As}(\mathrm{III})$ y As(V), se presentan en el cuadro IV.

CUADRO V. CONDICIONES EXPERIMENTALES DE LECTURA DE LAS MUESTRAS Y PATRONES POR MEDIO DE ICP-MS EN MODO DE ANÁLISIS CUANTITATIVO

\begin{tabular}{lcc}
\hline Parámetro & $\begin{array}{c}\text { Valor promedio } \\
\text { Empleado }\end{array}$ & $\begin{array}{c}\text { Valor recomendado } \\
\text { por el fabricante }\end{array}$ \\
\hline Temperatura de la cámara de espray de doble paso $\left({ }^{\circ} \mathrm{C}\right)$ & 2.0 & 2.0 \\
Poder de radiofrecuencia $(\mathrm{W})$ & 1550 & 1550 \\
Presión de análisis $(\mathrm{Pa})$ & $1.32 \times 10^{-4}$ & $1.1 \times 10^{-4}$ a $2.0 \times 10^{-4}$ \\
Presión de gas argón $(\mathrm{KPa})$ & 600 & $500 \mathrm{a} 700$ \\
Presión de gas helio $(\mathrm{KPa})$ & 100 & $90 \mathrm{a} 130$ \\
Flujo de gas argón $(\mathrm{L} / \mathrm{s})$ & 10.0 & 10.0 \\
Flujo de gas helio para el plasma $(\mathrm{L} / \mathrm{s})$ & 12.0 & 12.0 \\
Flujo gas helio de arrastre $(\mathrm{L} / \mathrm{s})$ & 1.0 & 0.8 a 1.3 \\
Flujo gas helio auxiliar $(\mathrm{L} / \mathrm{s})$ & 1.0 & $0 \mathrm{a} 1.0$ \\
Voltaje lente Extract $1(\mathrm{~V})$ & 0 & 0 \\
Voltaje lente Extract $2(\mathrm{~V})$ & -195.0 & -180.0 \\
Voltaje lente Omega bias $(\mathrm{V})$ & -80 & -80 \\
Voltaje lentes Omega $(\mathrm{V})$ & 10 & 10 \\
Velocidad bomba peristáltica $(\mathrm{rps})$ & 0.1 & 0.1 \\
\hline
\end{tabular}




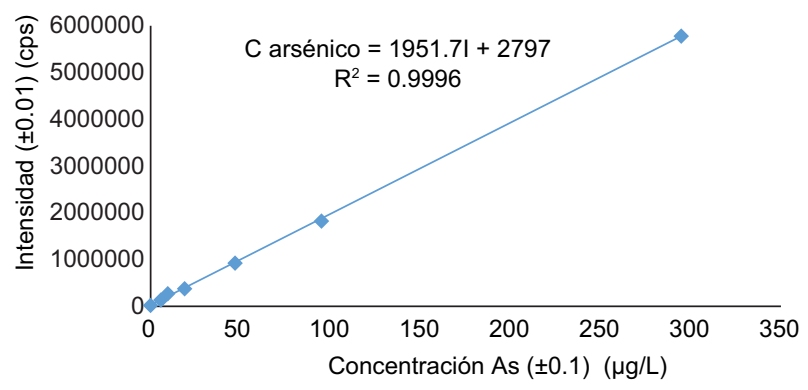

Fig. 1. Ejemplo de curva de calibración para la cuantificación de As(III) y As(V) expresado como arsénico total. cps: cuentas por segundo

Las recuperaciones de arsénico total se encontraron entre el 99 y el $104 \%$, la desviación estándar no supera los $7.6 \mu \mathrm{g} / \mathrm{L}$ y los CV oscilaron entre $1.1 \mathrm{y}$ $7.3 \%$.

El arsénico retenido en el filtro corresponde al coloidal o precipitado y el que permanece en disolución se denomina arsénico soluble o disuelto. En la literatura consultada se indica que no existe un método ideal para remover material particulado del agua, sin embargo, el empleo de filtros de $0.45 \mu \mathrm{m}$ de un uso único son ampliamente utilizados, ya que son de fácil adquisición y además no introducen contaminación en la muestra (McCleskey et al. 2004).

Dicha filtración también se efectuó con el fin de retirar turbiedad e impurezas que pueden afectar el desempeño de la resina y puedan obstruir los pequeños sitios de intercambio en el proceso de intercambio iónico. Las recuperaciones de arsénico soluble estuvieron en el ámbito entre el $99 \%$ y $101 \%$ con desviaciones estándar inferiores a $7.9 \mu \mathrm{g} / \mathrm{L}$ y CV inferiores al $7.8 \%$.

Respecto a las recuperaciones de $\mathrm{As}(\mathrm{V})$ en las distintas mezclas, sólo las de $5.0 \mu \mathrm{g} / \mathrm{L}$ en $\mathrm{As}(\mathrm{V})$ y de $50.0 \mu \mathrm{g} / \mathrm{L}$ en $\mathrm{As}(\mathrm{III})$ tuvieron la mayor dispersión en las recuperaciones del arsénico pentavalente, obteniéndose un CV de $12.8 \%$; sin embargo, el promedio de dichas recuperaciones fue de $103 \%$. Las otras recuperaciones de $\mathrm{As}(\mathrm{V})$ en las otras mezclas fueron de 97 y $103 \%$ con CV de 1.1 a $5.7 \%$.

En el caso de las recuperaciones de As(III), las mezclas de $\mathrm{As}(\mathrm{V}) / \mathrm{As}(\mathrm{III})$ de 25.0 y $5.0 \mu \mathrm{g} / \mathrm{L}, 0$ y $5.0 \mu \mathrm{g} / \mathrm{L}$, y 50.0 y $5.0 \mu \mathrm{g} / \mathrm{L}$, son las que presentaron mayor dispersión de arsénico trivalente (CV: 9.3, 10.8 y $8.4 \%$, respectivamente). Para el caso de estas tres mezclas, la concentración de arsenito es baja $(5.0 \mu \mathrm{g} / \mathrm{L})$, por lo que es posible que parte de éste se haya oxidado en el proceso de filtración y separación cromatográfica; por ende, que se obtuvieran recuperaciones tan variables (entre 88 y $117 \%$ ).

Cuando la concentración de As(III) en las mezclas fue más alta $(25.0 \mu \mathrm{g} / \mathrm{L}$ o $50.0 \mu \mathrm{g} / \mathrm{L})$, las recuperaciones fueron de 99 a $102 \%$, con una dispersión menor (CV entre $1.7 \%$ y $2.9 \%$ ). De forma general, la incertidumbre del método afectará mucho más a las concentraciones más bajas respecto a las más altas.

Para evaluar realmente el método se fortificaron cuatro muestras reales de agua natural sin arsénico (concentración no detectable). La fortificación se realizó a partir de mezclas de $\mathrm{As}(\mathrm{III}) / \mathrm{As}(\mathrm{V})$ a distintas concentraciones de materiales de referencia certificados para ICP-MS, ya que la pureza de la sal de As(III) no era adecuada para trabajar las recuperaciones en agua natural. Las recuperaciones de As(III) fueron de 99.6 a $102 \%$ mientras que para $\mathrm{As}(\mathrm{V})$ entre $99.2 \%$ a $104 \%$ (cuadro VI).

Por otro lado, se fortificó una quinta muestra de agua natural con presencia de $14.6 \mu \mathrm{g} / \mathrm{L}$ de arsénico, realizándose tres mezclas de $\mathrm{As}(\mathrm{III}) / \mathrm{As}(\mathrm{V})$ a distintas concentraciones. Se llevó a cabo un procedimiento de especiación de arsénico al agua natural y las concentraciones naturales de As(III)- s(V) en la fuente fueron de $10.2 \mu \mathrm{g} / \mathrm{L}$ de $\mathrm{As}(\mathrm{V})$ y $4.4 \mu \mathrm{g} / \mathrm{L}$ de

CUADRO VI. RESULTADO DE LAS RECUPERACIONES DE As(III)/As(V) EN AGUAS NATURALES SIN ARSÉNICO

\begin{tabular}{|c|c|c|c|c|c|c|}
\hline $\begin{array}{c}\text { Mezcla } \\
\text { As(III)/As(V) }\end{array}$ & $\begin{array}{l}\text { Concentración } \\
\text { teórica de } \\
\text { As }(\mathrm{III})(\mu \mathrm{g} / \mathrm{L})\end{array}$ & $\begin{array}{l}\text { Concentración } \\
\text { obtenida de } \\
\text { As(III) }(\mu \mathrm{g} / \mathrm{L})\end{array}$ & $\begin{array}{l}\text { Recuperación de } \\
\text { As(III) } \\
(\%)\end{array}$ & $\begin{array}{l}\text { Concentración de } \\
\qquad \begin{array}{c}\operatorname{As}(\mathrm{V}) \\
(\mu \mathrm{g} / \mathrm{L})\end{array}\end{array}$ & $\begin{array}{l}\text { Concentración } \\
\text { obtenida de } \\
\text { As }(V)(\mu \mathrm{g} / \mathrm{L})\end{array}$ & $\begin{array}{c}\text { Recuperación de } \\
\operatorname{As}(\mathrm{V}) \\
(\%)\end{array}$ \\
\hline 1 & 25.0 & 24.9 & 99.6 & 25.0 & 24.8 & 99.2 \\
\hline 2 & 5.0 & 5.1 & 102 & 50.0 & 49.9 & 99.8 \\
\hline 3 & 50.0 & 50.3 & 100.6 & 5.0 & 5.2 & 104 \\
\hline 4 & 25.0 & 25.2 & 100.8 & 0 & NA & NA \\
\hline
\end{tabular}

Incertidumbre de la concentración As: $\pm 0.1 \mu \mathrm{g} / \mathrm{L}$

NA: no aplica 
CUADRO VII. RESULTADO DE LAS RECUPERACIONES DE As(III)/As(V) EN AGUA NATURAL CON ARSÉNICO

\begin{tabular}{ccccccr}
\hline $\begin{array}{c}\text { Mezcla } \\
\text { As }(\mathrm{III}) \mathrm{y} \\
\text { As }(\mathrm{V})\end{array}$ & $\begin{array}{c}\text { Concentración } \\
\text { fortificada de } \\
\text { As(III) en la } \\
\text { muestra }(\mu \mathrm{g} / \mathrm{L})\end{array}$ & $\begin{array}{c}\text { Concentración } \\
\text { total obtenida de } \\
\text { As }(\mathrm{III})(\mu \mathrm{g} / \mathrm{L})\end{array}$ & $\begin{array}{c}\text { Recuperación de } \\
\text { As(III) } \\
\text { fortificado }(\%)\end{array}$ & $\begin{array}{c}\text { Concentración } \\
\text { fortificada de } \\
\text { As }(\mathrm{V}) \text { en la } \\
\text { muestra }(\mu \mathrm{g} / \mathrm{L})\end{array}$ & $\begin{array}{c}\text { Concentración } \\
\text { total obtenida de } \\
\text { As }(\mathrm{V})(\mu \mathrm{g} / \mathrm{L})\end{array}$ & $\begin{array}{c}\text { Recuperación de } \\
\text { As }(\mathrm{V}) \text { fortificado } \\
(\%)\end{array}$ \\
\hline 1 & 5.0 & 4.9 & 98 & 5.0 & 5.1 & 9.9 \\
2 & 10.0 & 10.1 & 101 & 10.0 & 5.2 & 99 \\
3 & 10.0 & 10.2 & 102 & 5.0 & 104 \\
\hline
\end{tabular}

Incertidumbre de la concentración As: $\pm 0.1 \mu \mathrm{g} / \mathrm{L}$

As(III). Las recuperaciones de As(III) fueron de 98 a $102 \%$ mientras que para $\mathrm{As}(\mathrm{V})$ de 99 a $104 \%$ (cuadro VII).

\section{CONCLUSIONES}

Las resinas de intercambio aniónico son una opción simple y económica. Poseen como ventaja la posibilidad de emplearse varias veces antes de que se saturen. La cromatografía de intercambio aniónico permite separar in situ el arsénico pentavalente del trivalente, evitando que estas dos especies puedan modificarse durante las etapas de transporte y posterior separación en el laboratorio.

Mediante la cuantificación en ICP-MS se obtuvieron bajos límites de detección y cuantificación, además de resultados exactos y precisos al evaluarse la repetibilidad y repetibilidad intermedia utilizando un material de referencia certificado de arsénico.

Con la metodología CIA/ICP-MS se lograron recuperaciones promedio de arsénico soluble superiores al $99 \%$ y de As(V) y As(III) mayores al $97 \%$. Cuando se trabajó a bajas concentraciones de arsenito o arseniato las recuperaciones fueron superiores a $88 \%$, en cualquiera de los dos estados de oxidación. Además, las recuperaciones de $\mathrm{As}(\mathrm{III})$ y $\mathrm{As}(\mathrm{V})$ en aguas naturales con presencia y ausencia de arsénico fueron bastante aceptables para evaluar la metodología.

\section{AGRADECIMIENTOS}

Las autoras extienden un sincero agradecimiento al personal del área de química de agua potable, área de química de aguas residuales y compañeros participantes del área de muestreo del Laboratorio Nacional de Aguas del Instituto Costarricense de Acueductos y Alcantarillados, por el apoyo brindado para la realización de este estudio.

\section{REFERENCIAS}

Agilent Technologies (2011). Agilent 7700 series ICP-MS mass hunter workstation user guide. Tokio, Japón, 58 pp.

Ahmed-Baig J., Gul-Kazi T., Qadir-Shah A., Abbas-Kandhro G., Imran-Afridi H., Balal-Arain M. y Jalbani N. (2010). Speciation and evaluation of arsenic in surface water and groundwater samples: a multivariate case study. Ecotox. Environ. Safe. 73 (5), 914-923.

DOI: 10.1016/j.ecoenv.2010.01.002

Akter K.F., Chen Z., Smith L., Davey D. y Naidu R. (2005). Speciation of arsenic in ground water samples: A comparative study of CE-UV, HG-AAS and LC-ICP-MS. Talanta 68 (2), 406-415.

DOI: $10.1016 /$ j.talanta.2005.09.011

APHA (2012). Standard methods for the examination of water and wastewater. 22a ed. American Public Health Association, Washington, EUA, 1325 pp.

Aposhian H.V. y Aposhian M.M. (2006). Arsenic toxicology: Five questions. Chem. Res. Toxicol. 19 (1), 1-13. DOI: $10.1021 / \mathrm{tx} 050106 \mathrm{~d}$

Bednar A.J., Garbarino J.R., Ranville J.F. y Wildeman T.R. (2002). Preserving the distribution of inorganic arsenic species in groundwater and acid mine drainage samples. Environ. Sci. Technol. 36, 2213-2218.

DOI: $10.1021 / \mathrm{es} 0157651$

Bednar A.J., Garbarino J.R., Burkhardt M.R., Ranville J.F. y Wildeman T.R. (2004). Field and laboratory arsenic speciation methods and their application to natural-water analysis. Water Res. 38 (2), 355-364. DOI: 10.1016/j.watres.2003.09.034

Currie L.A. (1995). Nomenclature in evaluation of analytical methods including detection and quantification capabilities (IUPAC Recommendations 1995). Pure Appl. Chem. 67 (10), 1699-1723.

DOI: $10.1351 /$ pac199567101699

Ellison S.L.R. y Williams A. (2012). EURACHEM/ CITAC guide: Quantifying uncertainty in analytical measurement. 3a ed. [en línea]. https://www.eurachem. org/images/stories/Guides/pdf/QUAM2012_P1.pdf $05 / 01 / 2015$ 
Haxton K. (2011). All about arsenic. Nat. Chem. 3 (9), 744. DOI: $10.1038 /$ nchem. 1118

Hagiwara K., Inui T., Koike Y., Aizawa M. y Nakamura T. (2015). Speciation of inorganic arsenic in drinking water by wavelength-dispersive X-ray fluorescence spectrometry after in situ preconcentration with miniature solid-phase extraction disks. Talanta 134, 739-744. DOI: 10.1016/j.talanta.2014.12.027

Kumar A.R. y Riyazuddin P. (2010). Preservation of inorganic arsenic species in environmental water samples for reliable speciation analysis. TrAC-Trend. Anal. Chem. 29 (10), 1212-1223.

DOI: $10.1016 /$ j.trac.2010.07.009

Litter M.I., Armienta M.A. y Farías S.S. (2009). Metodologías analíticas para la determinación y especiación de arsénico en aguas y suelos. IBEROARSEN (CYTED), Buenos Aires, 240 pp. [en línea]. https:// paginas.fe.up.pt/ cigar/html/documents/Monografia2_000.pdf 30/10/2014

McCleskey R.B., Nordstrom D.K. y Maest A.S. (2004). Preservation of water samples for arsenic (III/V) determinations: An evaluation of the literature and new analytical results. Appl. Geochem. 19 (7), 995-1009. DOI: 10.1016/j.apgeochem.2004.01.003

Miller J.N. y Miller J.C. (2002). Estadística y quimiometría para química analítica. 4a ed. Pearson Education. Madrid, España, 296 pp.
Moreno-Jiménez E. (2010). Recuperación de suelos mineros contaminados con arsénico mediante fitotecnologías. Tesis de Doctorado. Facultad de Ciencias, Universidad Autónoma de Madrid, Madrid, España, 208 pp.

Sharma V.K. y Sohn M. (2009). Aquatic arsenic: Toxicity, speciation, transformations, and remediation. Environ. Int. 35 (4), 743-759.

DOI: 10.1016/j.envint.2009.01.005

Smedley P.L. y Kinniburgh D.G. (2002). A review of the source, behaviour and distribution of arsenic in natural waters. Appl. Geochem. 17 (5), 517-568. DOI: $10.1016 / \mathrm{S} 0883-2927(02) 00018-5$

Sorg T.J., Chen A.S.C. y Wang L. (2014). Arsenic species in drinking water wells in the USA with high arsenic concentrations. Water Res. 48, (1) 156-169. DOI: $10.1016 /$ j.watres.2013.09.016

USEPA (2000). EPA/600/R-00/063. Arsenic removal from drinking water by coagulation-filtration and lime softening plants. Reporte. Office of Research and Development, United States Environmental Protection Agency, Washington, D.C., EUA, 112 pp.

Voice T.C., Flores-del Pino L.V, Havezov I. y Long D.T. (2011). Field deployable method for arsenic speciation in water. Phys. Chem. Earth. 36 (9-11), 436-441. DOI: $10.1016 /$ j.pce.2010.03.027

Wang J.S. y Wai C.M. (2004). Arsenic in drinking water. A global environmental problem. J. Chem. Edu. 81 (2), 207-213. DOI: 10.1021/ed081p20 